\title{
Chiral stationary phases based on chitosan bis(methylphenylcarbamate)-(isobutyrylamide) for high-performance liquid chromatography
}

\author{
Sheng Tang ${ }^{\mathrm{a}}$, Qin Bin ${ }^{\mathrm{a}}$, Wei Chen ${ }^{\mathrm{a}}$, Zheng-Wu Bai ${ }^{\mathrm{a}, *}$, Shao-Hua Huang ${ }^{\mathrm{b}}$ \\ a School of Chemistry and Environmental Engineering, Wuhan Institute of Technology, Wuhan 430073, China \\ ${ }^{\mathrm{b}}$ Key Laboratory of Biobased Materials, Qingdao Institute of Bioenergy and Bioprocess Technology, Chinese Academy of Sciences, Qingdao 266101, China
}

\section{A R T I C L E I N F O}

\section{Article history:}

Received 27 October 2015

Received in revised form 15 February 2016

Accepted 16 February 2016

Available online 19 February 2016

\section{Keywords:}

Chiral stationary phase

Chitosan derivatives

High-performance liquid chromatography

Chiral recognition

Enantiomer separation

\begin{abstract}
A B S T R A C T
A series of chitosan bis(methylphenylcarbamate)-(isobutyrylamide) derivatives were synthesized by carbamylating chitosan isobutyrylamide with different methylphenyl isocyanates. Then the prepared chitosan derivatives were coated onto 3-aminopropyl silica particles, resulting in a series of new chiral stationary phases (CSPs) for high-performance liquid chromatography. It was observed that the chiral recognition abilities of these coated-type CSPs depended very much on the substituents on the phenyl moieties of the chitosan derivatives, the eluent composition, as well as the structure of racemates. As a typical example, the eluent tolerance of the prepared CSP with the best enantioseparation ability was investigated in detail, and the results revealed that the CSP exhibited extraordinary solvent tolerance and could still work without significant loss in enantioseparation capability after being flushed with chloroform (100\%), ethyl acetate (100\%) and even THF/n-hexane (70/30, v/v), while the traditional coated-type CSPs based on the cellulose and amylose derivatives, such as cellulose tris(3,5dimethylphenylcarbamate) (CDMPC) and amylose tris(3,5-dimethylphenylcarbamate) (ADMPC), might be dissolved or highly swollen in these eluents. Therefore, the application of the resultant CSPs could address the problem of the dissolution and high swelling of traditional coated-type CSPs in some unusual eluents, broadening the possibility of eluent choice. In addition, a comparison of the prepared CSPs with the well known CDMPC- and ADMPC- based CSPs concerning the chiral recognition ability was also made. Separation performances achieved on the as-prepared CSPs in different eluents were found to be even superior to CDMPC-and ADMPC-based CSPs for the tested chiral compounds. In summary, we could safely draw the conclusion that the CSPs derived from chitosan isobutyrylamide derivatives were capable of excellent chiral recognition ability, and meanwhile possessed satisfactory eluent tolerance in a wider range of solvents.
\end{abstract}

(c) 2016 Elsevier B.V. All rights reserved.

\section{Introduction}

Owing to the availability of a wide range of chiral stationary phases (CSPs), enantioseparation by high-performance liquid chromatography (HPLC), as the most efficient and by far the most popular method for analyzing chiral compounds and obtaining optically pure isomers, has been advanced considerably in recent years [1-5]. Among the various CSPs, which have been developed for HPLC, polysaccharide-derived CSPs, especially those based on cellulose and amylose derivatives, have been most extensively studied and played significant roles in enantioseparation [6-10].

\footnotetext{
* Corresponding author. Tel.: +86 278719 5680; fax: +86 2787194560.

E-mail address: zwbai@wit.edu.cn (Z.-W. Bai).
}

This type of CSPs that appear to be most attractive exhibit excellent chiral recognition capabilities [11-17]. Some other polysaccharides, such as xylan, dextran, chitin and chitosan have also been reported as the starting material for the preparation of CSPs [18-21].

Chitosan is obtained by the deacetylation of chitin, which is one of the most abundant optically active biopolymers. Due to the fact that chitosan is conveniently modified at the hydroxyl and amino groups, chitosan derivatives have been used as the chiral selectors. Recently, Zhang et al. [22] developed some CSPs based on chitosan bis(phenylcarbamate)-(urea) derivatives for HPLC. The chiral recognition abilities of these coated-type CSPs significantly depended on the position and number of substituents on the phenyl groups. It was interestingly found that these chitosan derivatives based CSPs exhibited chiral resolution abilities for some 

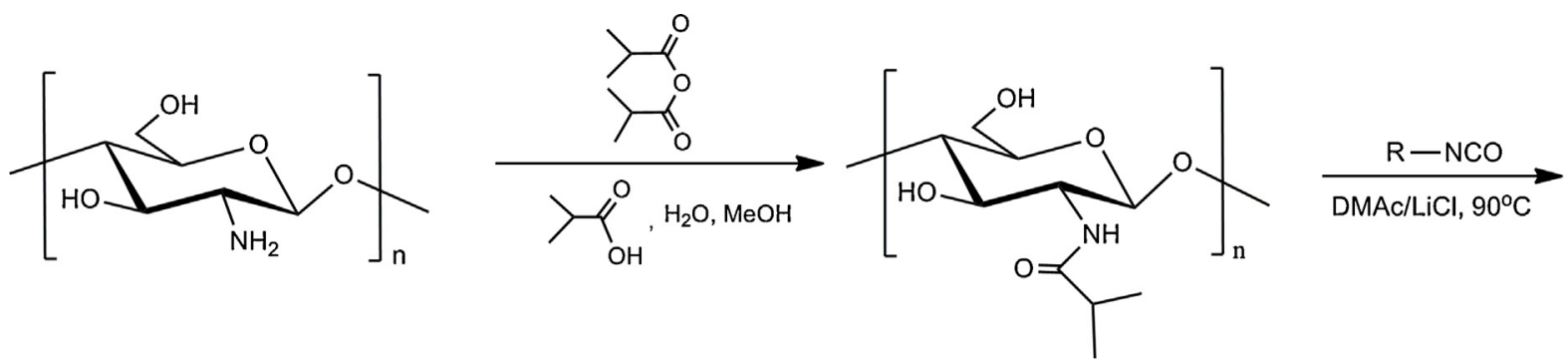<smiles>[R]NC(=O)OCC1C(OC(=O)N[R])C(OC(C)(C)C)C(NC(=O)C(C)C)C(C)(C)CC1(C)C</smiles>

P1-P6

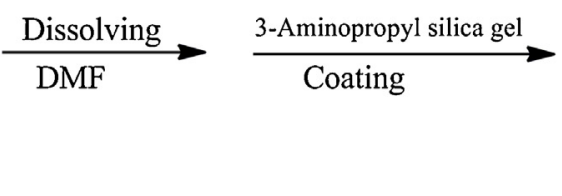<smiles>Cc1cc(C)cc(C)c1</smiles><smiles>[2H][AsH2][AsH2]</smiles><smiles>Cc1ccc(C)c(Cl)c1</smiles>

P5, CSP5: R=<smiles>Cc1cccc(C)c1</smiles>

P6, CSP6: $\mathrm{R}=$<smiles>Cc1ccccc1C</smiles>

Fig 1. Preparation scheme of chitosan derivatives and CSPs.

racemates that were equal to or even higher than those of cellulose or amylose derivatives based CSPs. Additionally, the solubility of chitosan derivatives in organic solvents was also found to be lower than that of conventional chiral selectors, such as cellulose tris(3,5-dimethylphenylcarbamate) (CDMPC) and amylose tris(3,5dimethylphenylcarbamate) (ADMPC). The lower solubility of chiral selectors is very important for the coated-type CSPs of polysaccharide derivatives, because these CSPs can work in a wider range of mobile phases, in which better enantioseparations may be gained. To date, there is still only a few studies and much less attention has been paid on chitosan derivatives based CSPs [23-31]. Meanwhile, most of the common coated-type CSPs exhibited poor solvent resistance in some organic solvents. Therefore, the development of new types of chitosan derivatives based CSPs, which not only are capable of effective chiral recognition but also possess desirable tolerance in a wider range of solvents, is of great significance.

In the present study, chitosan, which can be easily derivatized because of the presences of free amino and hydroxyl groups, was used as the starting material for the preparation of CSPs. Due to the fact that the reactivity of amino at 2-position and hydroxyl groups at 3- and 6-position of chitosan differs obviously, thus, different substituents, such as an alkyl group with relatively low electronic effect, can be introduced at 2-position of chitosan. In this work, a series of chitosan isobutyrylamide derivatives based CSPs were successfully prepared and their chiral separation performances were studied in detail. Then the solvent tolerance of the CSP with the best enantioseparation ability was investigated. A comparison of the obtained CSPs with the cellulose and amylose-derived CSPs, including the well known ones prepared from CDMPC and ADMPC, was also made. In comparison with the previously reported work [22], isobutyrylamide instead of phenyl urea was introduced at 2-position of chitosan in the present study. In addition, more systematic studies including the tolerance to various organic solvents, and the influence of eluent composition, as well as the structure of racemates on the enantioseparation of these newly developed CSPs, were reported.

\section{Experimental}

\subsection{Chemicals and apparatuses}

Spherical silica gel with a particle size of $7 \mu \mathrm{m}$ and a pore size of $1000 \AA$ was purchased from Daiso Co., Ltd. (Japan). 3Aminopropyltriethoxysilane was purchased from J\&K Scientific Ltd. (China). Chitin (from shrimp shell) was purchased from Hai Zhi Yuan Biological Products Co., Ltd. (China). Isobutyric anhydride (98\%) was purchased from Aladdin Reagent Co., Ltd. (China). Isocyanates (99\%) were purchased from Puyang Hongda Shengdao New Materials Co., Ltd. (China), Jinan Kaihua Chemical Co., Ltd. (China) and Dezhou Lübang Chemical Co., Ltd. (China). Isobutyric acid, $n$-amyl alcohol, $N, N$-dimethylacetamide (DMAc), 4dimethylaminopyridine (DMAP), $N, N$-dimethyl formamide (DMF) and other chemicals used for the preparation of chitosan derivatives or CSPs were analytical grade. These chemicals and the organic 


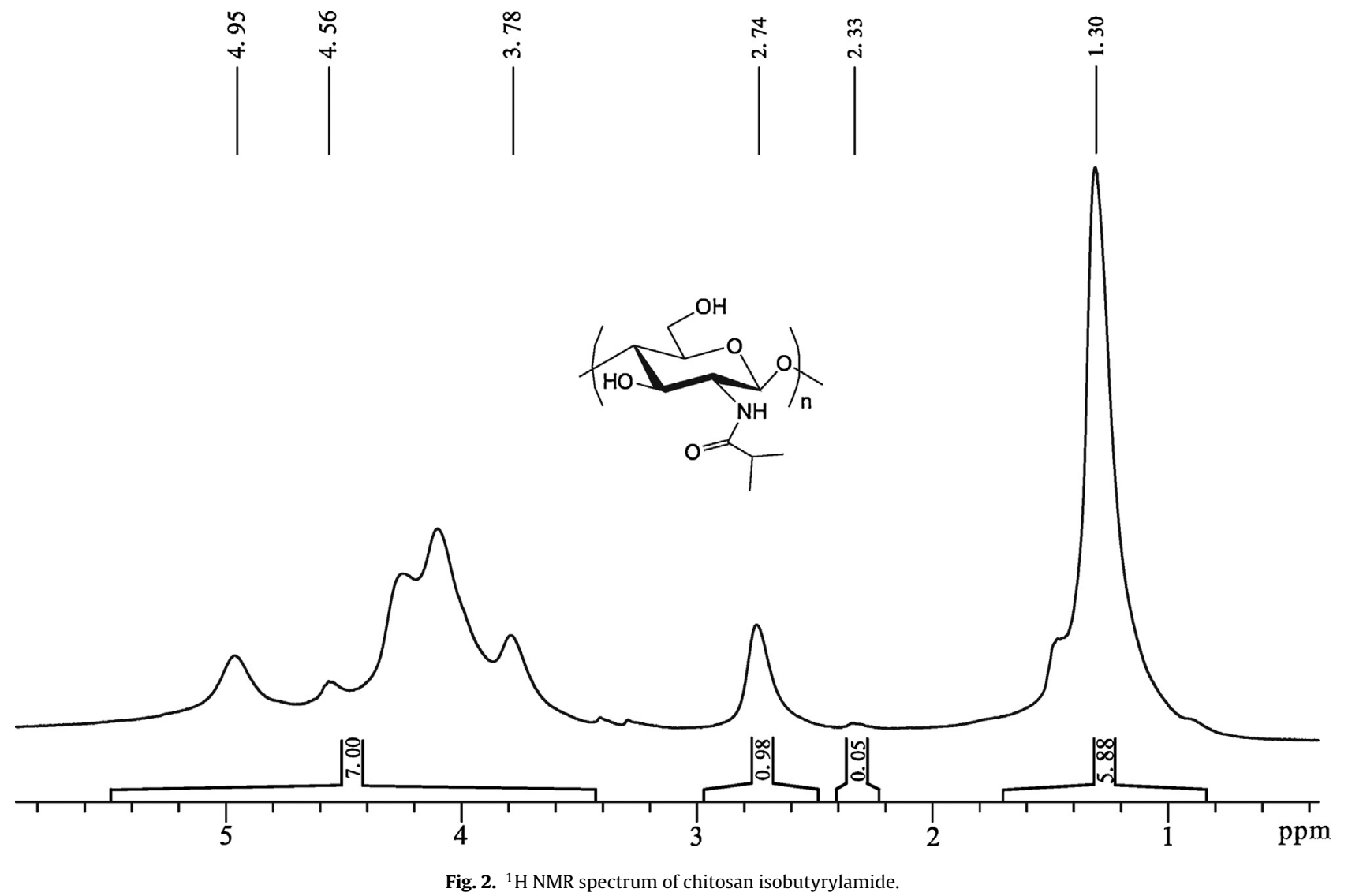

solvents used as chromatographic mobile phases were all purchased from Sinopharm Chemical Reagent Co., Ltd. (China).

IR spectra were recorded on a Nicolet FT-IR instrument (USA). ${ }^{1} \mathrm{H}$ NMR spectrum of chitosan or chitosan isobutyrylamide was measured by a $400 \mathrm{MHz}$ NMR spectrometer of Varian(USA) at $25^{\circ} \mathrm{C}$ with deuterated trifluoroacetic acid (TFA-d) as the solvent. ${ }^{1} \mathrm{H}$ NMR spectra of chitosan bis(methylphenylcarbamate)-(isobutyrylamide) were measured on a $600 \mathrm{MHz}$ NMR spectrometer of Bruker Avance III (Sweden) at $90^{\circ} \mathrm{C}$ with deuterated dimethyl sulfoxide (DMSO- $\mathrm{d}_{6}$ ) as the solvent. Elemental analysis (EA) measurement was conducted on an Elemental VarioEL III CHNOS apparatus (Germany). Stainless steel HPLC empty columns $(250 \times 4.6 \mathrm{~mm})$ were purchased from Hypersil (UK). The CSPs were packed into the stainless-steel columns by a slurry method with an Alltech Model 1666 slurry packer (USA). Chromatographic experiments were performed using a Waters HPLC system consisting of a photodiode array detector (Waters 996), a quaternary pump (Waters 600E), a thermostated column compartment and an autosampler (Waters $\left.717^{+}\right)$.

\subsection{Preparation of chitosan isobutyrylamide derivatives}

\subsubsection{Preparation of chitosan}

Chitin, $\mathrm{NaOH}$ and $n$-amyl alcohol (chitin/NaOH/n-amyl alcohol $=1 / 5 / 11$, mass ratio) were mixed. The resulting mixture was then stirred and refluxed at $150^{\circ} \mathrm{C}$ for $3 \mathrm{~h}$. After cooling down, the solid was filtered off, and then thoroughly washed with water to neutrality and dried yielding chitosan in a yield of $90 \%$.

\subsubsection{Preparation of chitosan isobutyrylamide}

$1.50 \mathrm{~g}$ of chitosan was dissolved in a solution of isobutyric acid $(0.75 \mathrm{~g})$ in $\mathrm{H}_{2} \mathrm{O}(30 \mathrm{ml})$, followed by adding methanol $(70 \mathrm{ml})$.
Then another solution of isobutyric anhydride $(12.5 \mathrm{~g})$ in methanol $(80 \mathrm{ml})$ was added to the reaction mixture, which was stirred mechanically for $7 \mathrm{~h}$ at ambient temperature. After the completion of the reaction, the mixture was poured into $500 \mathrm{ml}$ of $0.5 \mathrm{M}$ $\mathrm{KOH}-$ alcoholic solution and was stirred overnight. The solid was then filtered off and thoroughly washed with ethanol to neutrality. $2.02 \mathrm{~g}$ of chitosan isobutyrylamide was obtained in a yield of $89 \%$ after the treated solid was dried.

\subsubsection{Preparation of chitosan isobutyrylamide derivatives and CSPS}

$2.0 \mathrm{~g}$ of $\mathrm{LiCl}$ was placed in a flask, then $20 \mathrm{ml}$ of DMAc was added. The resulting mixture was heated to make $\mathrm{LiCl}$ dissolved completely and the temperature was kept at $80^{\circ} \mathrm{C}$. Then $1.44 \mathrm{~g}$ of chitosan isobutyrylamide was added and stirred for $24 \mathrm{~h} .3 .9 \mathrm{ml}$ of 4-methylphenyl isocyanate and a catalytic amount of DMAP were added and the reaction solution was stirred for $30 \mathrm{~h}$. After that, the solution was added dropwise into $200 \mathrm{ml}$ of methanol, and a white solid precipitated. The solid was filtered off, and was further purified by re-precipitation using DMF to dissolve it and methanol as precipitating agent. The re-precipitation was processed three times and the product was washed with methanol and water in sequence. The purified derivative was dried in vacuum, and $1.70 \mathrm{~g}$ of chitosan bis(4-methylphenylcarbamate)-(isobutyrylamide) (named as P1) was obtained. IR $\left(\mathrm{KBr}, \mathrm{cm}^{-1}\right) v$ : 3307(-NH), 2867( $\left.-\mathrm{CH}_{3}\right)$, 1730 (- $\left.\mathrm{CO}_{2}-\right), 1659$ (-CONH-), 3032-3078, 1604 (-Ph), 1534 $(-\mathrm{CONH}-,-\mathrm{Ph}) ;{ }^{1} \mathrm{H}$ NMR $(\delta, \mathrm{ppm}): 9.32-8.73(2 \mathrm{H}$, carbamate $\mathrm{H})$, 7.52-6.77 (9H, aromatic $\mathrm{H}$ and $\mathrm{C}_{2}$-amide $\mathrm{H}$ ), 5.13-3.27 (glucose unit skeleton $\mathrm{H}$, overlapped with $\left.\mathrm{H}_{2} \mathrm{O}\right), 2.37-1.88\left(7 \mathrm{H}, \mathrm{Ph}-\mathrm{CH}_{3}\right.$ and $\left.-\mathrm{CH}\left(\mathrm{CH}_{3}\right)_{2}\right), 1.02-0.52\left(6 \mathrm{H},-\mathrm{CH}\left(\mathrm{CH}_{3}\right)_{2}\right)$; Elemental analysis (EA)\%: Calcd $\left(\mathrm{C}_{26} \mathrm{H}_{31} \mathrm{~N}_{3} \mathrm{O}_{7} \cdot 0.50 \mathrm{H}_{2} \mathrm{O}\right)_{\mathrm{n}} \mathrm{C}: 61.65, \mathrm{H}: 6.37, \mathrm{~N}: 8.30$; Found $\mathrm{C}$ : 61.58, H: 6.50, N: 8.20 . 


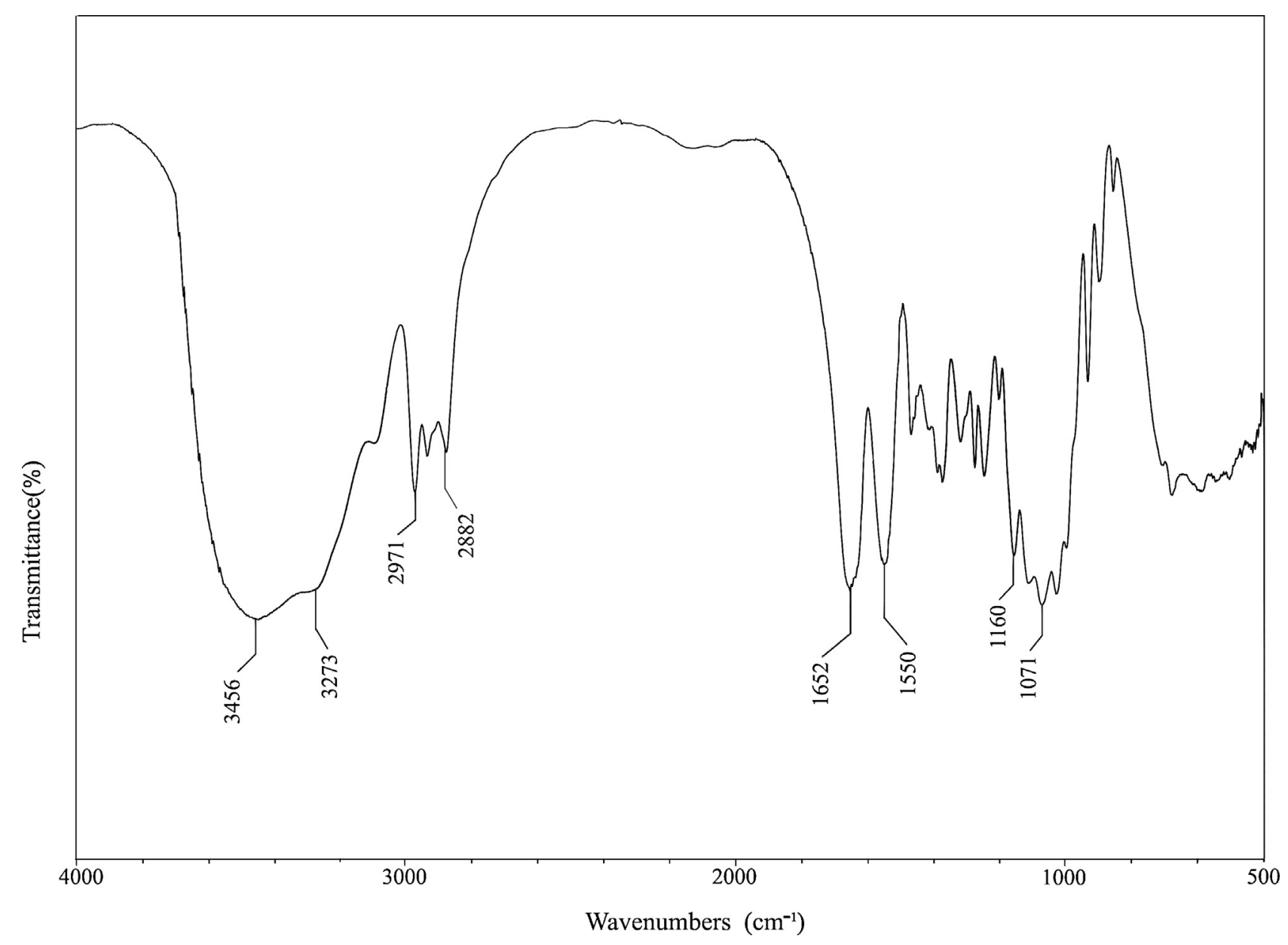

Fig. 3. IR spectrum of chitosan isobutyrylamide.

According to the above preparation methods, a series of chitosan isobutyrylamide derivatives were synthesized with different isocyanates as the modifiers (Fig. 1):

Chitosan bis(3,5-dimethylphenylcarbamate)-(isobutyrylamide) (P2). IR ( $\left.\mathrm{KBr}, \mathrm{cm}^{-1}\right) v$ : 3311 $(-\mathrm{NH}), 2869\left(-\mathrm{CH}_{3}\right), 1723\left(-\mathrm{CO}_{2}-\right)$, 1662 (-CONH-), 3017-3090, 1617 (-Ph), 1550 (-CONH-, - Ph); ${ }^{1} \mathrm{H}$ NMR ( $\left.\delta, \mathrm{ppm}\right)$ : 9.28-8.25 (2H, carbamate $\left.\mathrm{H}\right), 7.31-6.77$ $\left(5 \mathrm{H}\right.$, aromatic $\mathrm{H}$ and $\mathrm{C}_{2}$-amide $\left.\mathrm{H}\right), 6.74-6.34(2 \mathrm{H}$, aromatic $\mathrm{H})$, 5.19-3.26 $(7 \mathrm{H}$, glucose unit skeleton $\mathrm{H}), 2.34-1.71(13 \mathrm{H}$, $\mathrm{Ph}-\mathrm{CH}_{3}$ and $\left.-\mathrm{CH}\left(\mathrm{CH}_{3}\right)_{2}\right), 1.13-0.36\left(6 \mathrm{H},-\mathrm{CH}\left(\mathrm{CH}_{3}\right)_{2}\right)$; EA\%: Calcd $\left(\mathrm{C}_{28} \mathrm{H}_{35} \mathrm{~N}_{3} \mathrm{O}_{7} \cdot 0.50 \mathrm{H}_{2} \mathrm{O}\right)_{n} \mathrm{C}: 62.91, \mathrm{H}: 6.79, \mathrm{~N}: 7.86$; Found C: 63.03, $\mathrm{H}: 6.94$, N: 7.99.

Chitosan bis(3-chloro-4-methylphenylcarbamate)-(isobutyrylamide) (P3). IR ( $\left.\mathrm{KBr}, \mathrm{cm}^{-1}\right)$ v: 3295(-NH), 2869(- $\left.\mathrm{CH}_{3}\right), 1729$ $\left(-\mathrm{CO}_{2}-\right), 1662$ (-CONH-), 3030-3103, 1611 (-Ph), 1531 (-CONH-, $-\mathrm{Ph}) ;{ }^{1} \mathrm{H}$ NMR $(\delta, \mathrm{ppm}): 9.64-8.87$ (2H, carbamate $\mathrm{H})$, 7.71-6.76 $\left(7 \mathrm{H}\right.$, aromatic $\mathrm{H}$ and $\mathrm{C}_{2}$-amide $\left.\mathrm{H}\right), 5.24-3.26(7 \mathrm{H}$, glucose unit skeleton $\mathrm{H}), 2.36-1.85\left(7 \mathrm{H}, \mathrm{Ph}-\mathrm{CH}_{3}\right.$ and $\left.-\mathrm{CH}\left(\mathrm{CH}_{3}\right)_{2}\right)$, 0.99-0.50 (6H, $\left.-\mathrm{CH}\left(\mathrm{CH}_{3}\right)_{2}\right)$; EA\%: Calcd $\left(\mathrm{C}_{26} \mathrm{H}_{29} \mathrm{Cl}_{2} \mathrm{~N}_{3} \mathrm{O}_{7} \cdot 0.25 \mathrm{H}_{2} \mathrm{O}\right)_{n}$ C: 54.70, H: 5.21, N: 7.36; Found C: 54.84, H: 5.50, N: 7.34.

Chitosan bis(phenylcarbamate)-(isobutyrylamide) (P4). IR $\left(\mathrm{KBr}, \mathrm{cm}^{-1}\right) \quad v$ : 3414(-NH), $1723\left(-\mathrm{CO}_{2}-\right), 1662$ (-CONH-), 3036-3087, $1598(-\mathrm{Ph}), 1543(-\mathrm{CONH}-,-\mathrm{Ph}) ;{ }^{1} \mathrm{H}$ NMR $(\delta$, ppm): 9.47-8.67 (2H, carbamate $\mathrm{H}), 7.63-6.61(11 \mathrm{H}$, aromatic $\mathrm{H}$ and $\mathrm{C}_{2}$-amide $\left.\mathrm{H}\right), 5.17-3.23(7 \mathrm{H}$, glucose unit skeleton $\mathrm{H})$, 2.32-1.98 $\left(1 \mathrm{H},-\mathrm{CH}\left(\mathrm{CH}_{3}\right)_{2}\right), 1.00-0.53\left(6 \mathrm{H},-\mathrm{CH}\left(\mathrm{CH}_{3}\right)_{2}\right)$; EA\%: Calcd $\left(\mathrm{C}_{24} \mathrm{H}_{27} \mathrm{~N}_{3} \mathrm{O}_{7} \cdot 1.25 \mathrm{H}_{2} \mathrm{O}\right)_{\mathrm{n}} \mathrm{C}: 58.59, \mathrm{H}: 6.04, \mathrm{~N}: 8.54$; Found $\mathrm{C}$ : 58.57, H: 6.34, N: 8.67.
Chitosan bis(3-methylphenylcarbamate)-(isobutyrylamide) (P5). IR ( $\left.\mathrm{KBr}, \mathrm{cm}^{-1}\right) v$ : 3407(-NH), 2869 $\left(-\mathrm{CH}_{3}\right), 1726\left(-\mathrm{CO}_{2}-\right)$, 1659 (-CONH-), 3030-3097, 1614 (-Ph), 1547 (-CONH-, -Ph); ${ }^{1} \mathrm{H}$ NMR $(\delta, \mathrm{ppm}): 9.42-8.71(2 \mathrm{H}$, carbamate $\mathrm{H}), 7.53-6.54(9 \mathrm{H}$, aromatic $\mathrm{H}$ and $\mathrm{C}_{2}$-amide $\left.\mathrm{H}\right), 5.20-3.22(7 \mathrm{H}$, glucose unit skeleton $\mathrm{H}), 2.36-1.95\left(7 \mathrm{H}, \mathrm{Ph}-\mathrm{CH}_{3}\right.$ and $\left.-\mathrm{CH}\left(\mathrm{CH}_{3}\right)_{2}\right), 1.07-0.50(6 \mathrm{H}$, $\left.-\mathrm{CH}\left(\mathrm{CH}_{3}\right)_{2}\right)$; EA\%: Calcd $\left(\mathrm{C}_{26} \mathrm{H}_{31} \mathrm{~N}_{3} \mathrm{O}_{7} \cdot 0.5 \mathrm{H}_{2} \mathrm{O}\right)_{\mathrm{n}} \mathrm{C}: 61.65, \mathrm{H}: 6.37$, $\mathrm{N}: 8.30$; Found C: 61.85, H: 6.47, N: 8.48.

Chitosan bis(2-methylphenylcarbamate)-(isobutyrylamide) (P6). IR ( $\left.\mathrm{KBr}, \mathrm{cm}^{-1}\right)$ v: 3427(-NH), 2869 $\left(-\mathrm{CH}_{3}\right), 1723\left(-\mathrm{CO}_{2}-\right)$, 1656 (-CONH-), 3020-3065, 1588 (-Ph), 1527 (-CONH-, -Ph); ${ }^{1} \mathrm{H}$ NMR $(\delta, \mathrm{ppm})$ : 8.63-8.19 $(1 \mathrm{H}$, carbamate $\mathrm{H}), 8.13-7.56$ $(1 \mathrm{H}$, carbamate $\mathrm{H}), 7.52-6.79\left(9 \mathrm{H}\right.$, aromatic $\mathrm{H}$ and $\mathrm{C}_{2}$-amide $\mathrm{H}), 5.18-3.33(7 \mathrm{H}$, glucose unit skeleton $\mathrm{H}), 2.33-1.91(7 \mathrm{H}$, $\mathrm{Ph}-\mathrm{CH}_{3}$ and $\left.-\mathrm{CH}\left(\mathrm{CH}_{3}\right)_{2}\right), 1.05-0.65\left(6 \mathrm{H},-\mathrm{CH}\left(\mathrm{CH}_{3}\right)_{2}\right)$; EA\%: Calcd $\left(\mathrm{C}_{26} \mathrm{H}_{31} \mathrm{~N}_{3} \mathrm{O}_{7} \cdot 0.25 \mathrm{H}_{2} \mathrm{O}\right)_{n} \mathrm{C}: 62.20, \mathrm{H}: 6.32, \mathrm{~N}: 8.37$; Found C: 62.14 , $\mathrm{H}: 6.63, \mathrm{~N}: 7.94$.

3-Aminopropyl silica gel was prepared with dried silica gel and 3-aminopropyltriethoxysilane in toluene [32]. P1 (0.65 g) was dissolved in DMF $(30 \mathrm{ml})$. Then 3-aminopropyl silica gel $(2.62 \mathrm{~g})$ was added into the above solution $(10 \mathrm{ml})$, and the solvent was removed on a rotation evaporator under vacuum. This coating process was then repeated twice. After drying in vacuum, a white powder (named as CSP1) were obtained. The chiral selector was fed at a ratio of about $20 \%$ by weight.

According to the above coating method, a series of CSPs were prepared when different chitosan bis(methylphenylcarbamate)(isobutyrylamide) derivatives were used as the chiral selectors (Fig. 1). The ADMPC- and CDMPC-based CSPs (named as CSPa and 


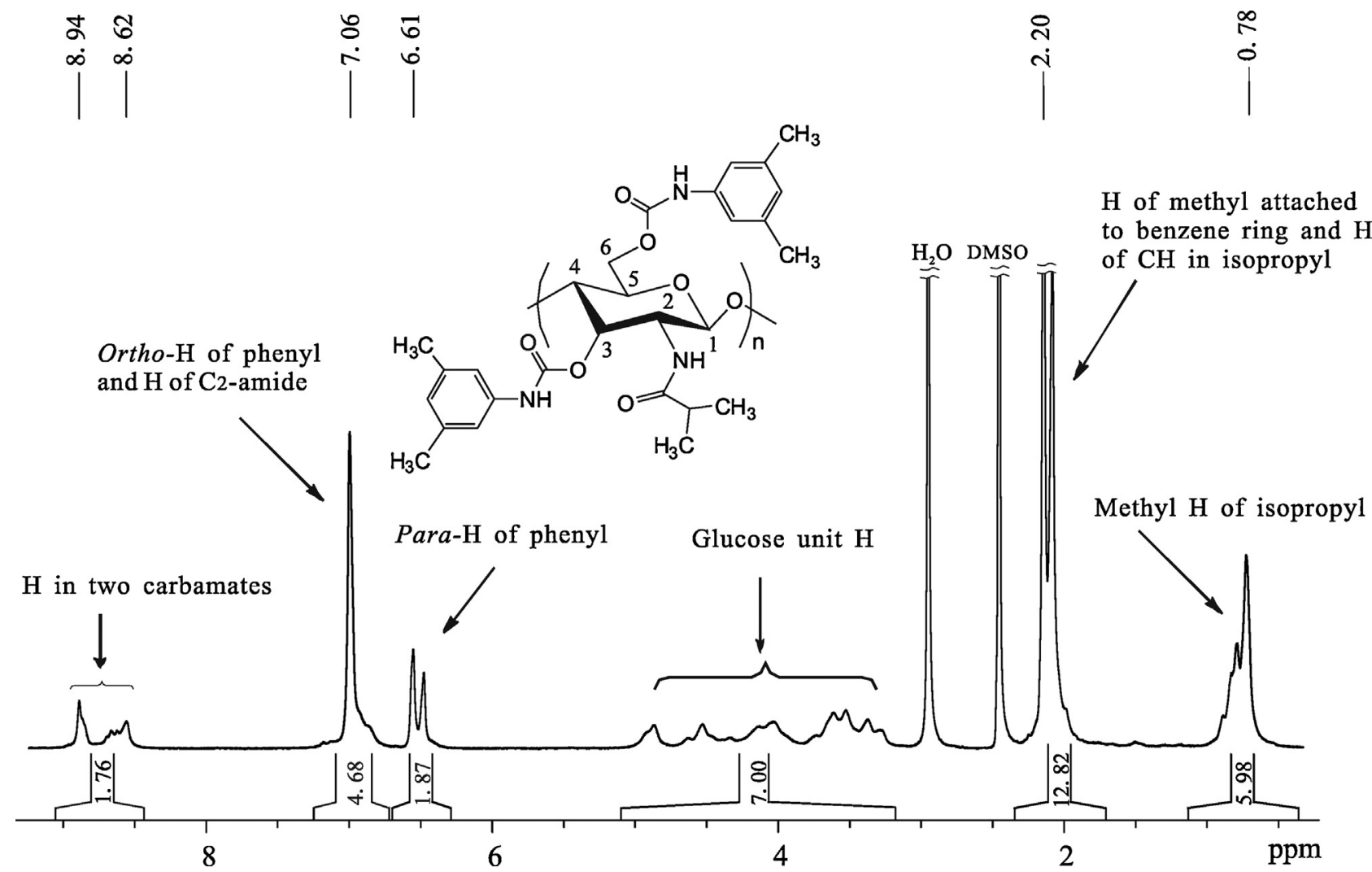

Fig. 4. ${ }^{1} \mathrm{H}$ NMR spectrum of chitosan bis(3,5-dimethylphenylcarbamate)-(isobutyrylamide).

$\mathrm{CSPb}$, respectively) were prepared in our previous work with the same support and coating method to prepare CSPs1-6 [7]. ADMPC and CDMPC were also fed at a ratio of $20 \%$ by weight.

\subsection{Column packing and chromatography}

CSPs1-6 were, respectively, suspended in a solution consisting of $n$-hexane and isopropanol ( $n$-hexane/isopropanol, 90/10, $\mathrm{v} / \mathrm{v}$, the same definition for other cases hereafter). The suspensions were sonicated to form slurries, and then were packed into empty stainless-steel columns with $n$-hexane as the displacer solvent [7]. The columns of CSPa and CSPb were packed with the same way. After packing, the columns were flushed with $n$-hexane/isopropanol (90/10) till the column pressure and baseline were stable. In this mobile phase, the dead times $\left(t_{0}\right)$ were determined with 1,3,5-tris-(tert-butyl) benzene, and each column efficiency was determined by using $1,1^{\prime}$-biphenyl as the test solute. The enantioseparation results were obtained under the same conditions for all the CSPs.

Enantioseparation evaluations were performed in the mobile phases of $n$-hexane/isopropanol (90/10), $n$-hexane/ethanol (90/10) and $n$-hexane/ethanol/methanol (90/5/5). All of the mobile phases were filtered and degassed before use. The chiral sample solutions were prepared by dissolving the analytes in ethanol $\left(1 \mathrm{mg} \mathrm{ml}^{-1}\right)$ and were filtered through $0.2 \mu \mathrm{m}$ filters. The injection volume was $10 \mu \mathrm{l}$. The column temperature was set at $25^{\circ} \mathrm{C}$, and the flow rate was $1 \mathrm{ml} \mathrm{min}^{-1}$ for the detection of all analytes. All analytes were measured at the wavelength where they absorb most. Retention factor $(k)$ was calculated as $\left(t_{R}-t_{0}\right) / t_{0}$, where $t_{R}$ represents the retention time of the analytes. Separation factor $(\alpha)$ was calculated as the ratio of the retention factors of two enantiomers $\left(k_{2} / k_{1}\right)$. Resolution $\left(R_{\mathrm{S}}\right)$ was evaluated by using the following equation:
$R_{\mathrm{S}}=2\left(t_{\mathrm{R}(2)}-t_{\mathrm{R}(1)}\right) /\left(w_{1}+w_{2}\right)$, where $w_{1}$ and $w_{2}$ are the peak width of chromatographic peaks of two enantiomers, respectively. The elution order of the chiral analytes was determined by varying the injection amount of a single enantiomer (if available) or by a PDR-Chemical LLC polarimeter (USA), in which the concentration of sample solutions was increased to $10 \mathrm{mg} \mathrm{ml}^{-1}$.

\section{Results and discussion}

\subsection{Characterization of the chitosan derivatives}

In this work, chitosan was prepared by the deacetylation of chitin in $n$-amyl alcohol. The degree of deacetylation (D.D.) amounted to $98 \%$, which was estimated by ${ }^{1} \mathrm{H}$ NMR (Fig. S1) $[33,34]$. The structure of chitosan isobutyrylamide was confirmed by its ${ }^{1} \mathrm{H}$ NMR spectrum (Fig. 2), in which the D.D. was found to be $98.3 \%$. Because the residual acetyl in chitosan isobutyrylamide is very little, the ratios of integration areas match well with the structure of chitosan isobutyrylamide. Besides, Fig. 3 shows the IR spectrum of the prepared chitosan isobutyrylamide, in which no peak corresponding to ester group can be observed, thus substantiating that isobutyryl group is connected with amino group rather than hydroxyl group in glucose unit. The ${ }^{1} \mathrm{H}$ NMR spectrum of chitosan bis(3,5-dimethylphenylcarbamate)-(isobutyrylamide) is exhibited in Fig. 4, and the ${ }^{1} \mathrm{H}$ NMR spectra of the rest chitosan isobutyrylamide derivatives are presented in Figs. S2-S6. As shown in Fig. 4, the ratios of integration areas could also match the desired structure of chitosan bis(3,5-dimethylphenylcarbamate)(isobutyrylamide). Therefore, the hydroxyl groups at 3- and 6-positions of chitosan isobutyrylamide were almost completely carbamylated. During purification, P1-P6 were re-precipitated three times at least and as a result, the polymers bearing residual 
<smiles>Cc1ccc2c(c1)CN1CCN2Cc2ccc(C)cc21</smiles>

1

troger's base<smiles>O=C1NC(c2ccccc2)CO1</smiles>

6

4-phenyloxazolidin2-one<smiles>O=C1CC(c2ccccc2)Oc2ccccc21</smiles>

2<smiles>CC(O)c1ccc2ccccc2c1</smiles>

3<smiles>CS(=O)(=O)c1ccccc1</smiles>

4<smiles>CC(O)c1ccccc1</smiles>

5 1-(2-naphthyl)-ethanol methyl phenyl 1-phenylethanol sulf oxide<smiles>Cc1ccc(C(=O)NC(C)c2ccccc2)cc1</smiles>

9<smiles>O=C(c1ccccc1)C(O)c1ccccc1</smiles>

10<smiles>COc1ccc(C(C)NC(=O)Nc2ccccc2)cc1</smiles>

11

benzoin 1-(1-(4-methoxyphenyl) ethyl)-3-phenylurea 1-(1-phenylethyl)- 1-(2,4-dichlorophenyl)-2- 4-methyl- $N$-(13-(p-tolyl)urea<smiles>COc1ccc(C(C)NC(=O)c2cc([N+](=O)[O-])cc([N+](=O)[O-])c2)cc1</smiles>

$N$-(1-(4-methoxyphenyl) ethyl)-3,5-dinitrobenzamide
13 glutethimide<smiles>CN(CBr)CCCC1(c2ccc(F)cc2)OCc2cc(C#N)ccc21</smiles>

14

citalopram hydrobromide<smiles>O=C1Nc2ccc(Cl)cc2C(F)(C(F)(F)F)O1</smiles>

15

ef avirenz<smiles>COc1ccc2[nH]c(S(=O)Cc3ncc(C)c(OC)c3C)nc2c1</smiles>

17

omeprazole sodium<smiles>CN(C)CCCC(O)(c1ccc(F)cc1)c1ccc(C#N)cc1CO</smiles>

18

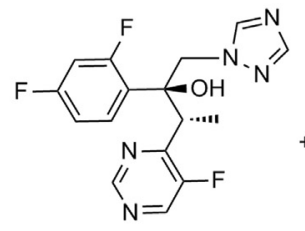

(2S,3R)<smiles>C[C@H](c1ncncc1F)[C@@](O)(Cn1cncn1)c1ccc(F)cc1F</smiles>

$(2 \mathrm{R}, 3 \mathrm{~S})$ 4-(4-(dimethylamino)-1-(4-fluorophenyl)-1hydroxybutyl)-3-(hydroxymethyl)benzonitrile voriconazole's enantiomer voriconazole

Fig. 5. Structures of the tested chiral analytes.

a

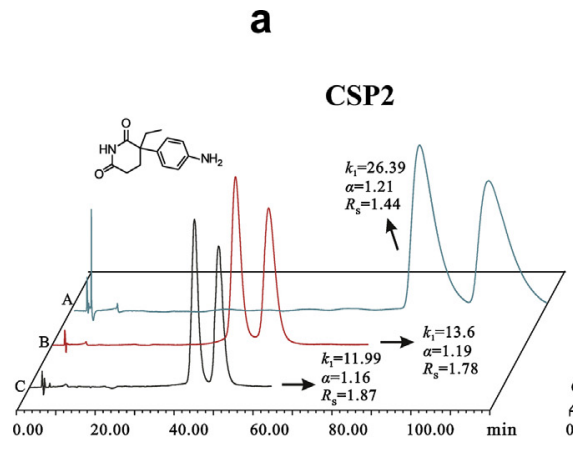

b

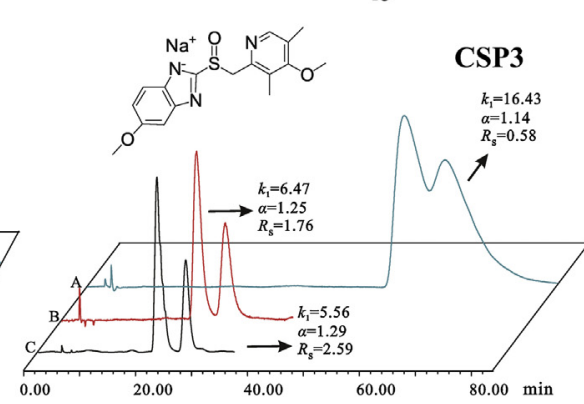

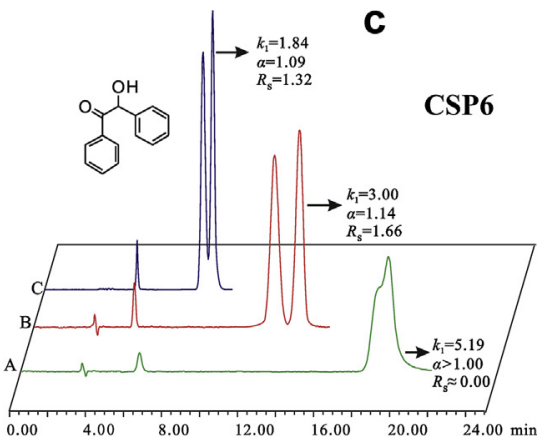

Eluents: (A) n-hexane/isopropanol; (B) n-hexane/ethanol; (C) n-hexane/methanol/ethanol

Fig. 6. Chromatograms for the resolution of racemate $\mathbf{1 2}$ on CSP2 (a), the resolution of racemate $\mathbf{1 7}$ on CSP3 (b) and the resolution of racemate $\mathbf{1 0}$ on CSP6 (c).

acetyl group became less and less. Thus, the calculated values of EA corresponded to completely deacetylated P1-P6. Because P1-P6 are hygroscopic at various extent and they were finally washed by water when purified, it was extremely difficult to fully remove the adsorbed water.

\subsection{Evaluation of chiral recognition ability of CSPS}

All these six prepared CSPs exhibited adequate column efficiencies, which were in the range from 28000 to 34000 plates per meter, indicating that they were all suitable to be used as the chromatographic stationary phases for enantioseparation. 
Table 1

Enantioseparation evaluation of CSPs1-6.

\begin{tabular}{|c|c|c|c|c|c|c|c|c|c|c|c|c|c|c|c|c|c|c|c|}
\hline \multirow[t]{2}{*}{ S.N. } & \multicolumn{3}{|l|}{ CSP1 } & \multicolumn{3}{|l|}{ CSP2 } & \multicolumn{3}{|l|}{ CSP3 } & \multicolumn{3}{|l|}{ CSP4 } & \multicolumn{3}{|l|}{ CSP5 } & \multicolumn{3}{|l|}{ CSP6 } & \multirow[t]{2}{*}{ M.P. } \\
\hline & $k_{1}$ & $\alpha$ & $R_{\mathrm{S}}$ & $k_{1}$ & $\alpha$ & $R_{\mathrm{S}}$ & $k_{1}$ & $\alpha$ & $R_{\mathrm{S}}$ & $k_{1}$ & $\alpha$ & $R_{\mathrm{S}}$ & $k_{1}$ & $\alpha$ & $R_{\mathrm{S}}$ & $k_{1}$ & $\alpha$ & $R_{\mathrm{S}}$ & \\
\hline \multirow[t]{3}{*}{1} & $0.37^{-}$ & 1.26 & 1.33 & $0.28^{-}$ & 1.40 & 1.58 & $0.31^{-}$ & 1.21 & 0.83 & $0.46^{-}$ & 1.46 & 1.98 & $0.46^{-}$ & 1.54 & 2.40 & 0.34 & 1.00 & 0.00 & A \\
\hline & $0.30^{-}$ & 1.36 & 1.61 & $0.24^{-}$ & 1.32 & 1.08 & $0.26^{-}$ & 1.24 & 0.95 & $0.30^{-}$ & 1.43 & 1.70 & $0.32^{-}$ & 1.49 & 1.90 & $0.38^{-}$ & 1.14 & 0.42 & B \\
\hline & $0.33^{-}$ & 1.32 & 1.60 & $0.29^{-}$ & 1.39 & 1.47 & $0.30^{-}$ & 1.22 & 0.87 & $0.29^{-}$ & 1.44 & 1.72 & $0.32^{-}$ & 1.51 & 1.94 & 0.28 & 1.00 & 0.00 & $C$ \\
\hline \multirow[t]{3}{*}{2} & $1.00^{-}$ & 1.20 & 1.94 & $0.96^{-}$ & 1.36 & 2.96 & $1.23^{-}$ & 1.47 & 4.19 & $1.54^{-}$ & 1.21 & 1.90 & $1.51^{-}$ & 1.31 & 2.64 & $1.24^{-}$ & 1.07 & 0.17 & A \\
\hline & $0.78^{-}$ & 1.29 & 2.63 & $0.78^{-}$ & 1.31 & 2.38 & $0.96^{-}$ & 1.43 & 3.74 & $0.91^{-}$ & 1.25 & 2.19 & $1.06^{-}$ & 1.34 & 2.81 & $1.19^{-}$ & 1.08 & 0.77 & B \\
\hline & $0.90^{-}$ & 1.26 & 2.69 & $1.01^{-}$ & 1.34 & 2.70 & $1.17^{-}$ & 1.46 & 4.05 & $0.90^{-}$ & 1.26 & 2.24 & $1.04^{-}$ & 1.35 & 2.86 & 0.71 & 1.00 & 0.00 & C \\
\hline 3 & $1.93^{-}$ & 1.06 & 0.66 & 1.34 & 1.00 & 0.00 & $1.06^{-}$ & 1.07 & 0.50 & $2.31^{-}$ & 1.04 & 0.20 & 2.27 & 1.00 & 0.00 & 1.65 & 1.00 & 0.00 & A \\
\hline & $0.84^{-}$ & 1.07 & 0.42 & $0.77^{-}$ & 1.06 & 0.21 & $0.58^{-}$ & 1.06 & 0.16 & $0.71^{-}$ & 1.06 & 0.22 & $0.88^{-}$ & 1.03 & 0.10 & 0.95 & 1.00 & 0.00 & B \\
\hline & 0.91 & 1.00 & 0.00 & 0.85 & 1.00 & 0.00 & $0.88^{-}$ & 1.09 & 0.48 & 0.63 & 1.00 & 0.00 & 0.76 & 1.00 & 0.00 & 0.77 & 1.00 & 0.00 & C \\
\hline 4 & $3.30^{+}$ & 1.18 & 2.27 & $2.22^{+}$ & 1.15 & 1.77 & $5.18^{+}$ & 1.29 & 3.56 & $7.63^{+}$ & 1.20 & 1.93 & $5.93^{+}$ & 1.23 & 1.90 & 6.48 & 1.00 & 0.00 & A \\
\hline & $1.65^{+}$ & 1.22 & 2.37 & $1.38^{+}$ & 1.14 & 1.38 & $2.41^{+}$ & 1.29 & 3.54 & $2.47^{+}$ & 1.22 & 2.55 & $2.22^{+}$ & 1.22 & 2.42 & 3.26 & 1.00 & 0.00 & B \\
\hline & $1.70^{+}$ & 1.17 & 2.24 & $2.34^{+}$ & 1.14 & 1.49 & $4.02^{+}$ & 1.31 & 3.76 & $2.31^{+}$ & 1.23 & 2.69 & $2.11^{+}$ & 1.23 & 2.49 & 1.71 & 1.00 & 0.00 & C \\
\hline 5 & $0.75^{-}$ & 1.12 & 0.62 & $0.68^{-}$ & 1.08 & 0.21 & $0.67^{-}$ & 1.18 & 1.20 & $1.14^{-}$ & 1.12 & 1.14 & $1.09^{-}$ & 1.12 & 1.00 & $0.97^{-}$ & 1.05 & 0.15 & A \\
\hline & 0.54 & 1.07 & 0.12 & 0.52 & 1.00 & 0.00 & $0.44^{-}$ & 1.06 & 0.13 & $0.53^{-}$ & 1.05 & 0.10 & $0.58^{-}$ & 1.05 & 0.11 & 0.68 & 1.00 & 0.00 & B \\
\hline & 0.68 & 1.00 & 0.00 & $0.62^{-}$ & 1.07 & 0.20 & $0.53^{-}$ & 1.18 & 1.17 & 0.47 & 1.00 & 0.00 & 0.50 & 1.00 & 0.00 & 0.59 & 1.00 & 0.00 & C \\
\hline 6 & $10.53^{R}$ & 1.22 & 2.07 & $8.43^{\mathrm{R}}$ & 1.44 & 3.46 & $14.07^{\mathrm{R}}$ & 1.20 & 2.04 & Retentiont & ime $>1$ & $20 \mathrm{~min}$ & $21.14^{\mathrm{R}}$ & 1.20 & 1.78 & $19.74^{\mathrm{S}}$ & 1.32 & 2.20 & A \\
\hline & $5.66^{\mathrm{R}}$ & 1.31 & 3.76 & $5.18^{\mathrm{R}}$ & 1.35 & 3.32 & $7.34^{\mathrm{R}}$ & 1.12 & 1.52 & 7.28 & 1.00 & 0.00 & $7.45^{\mathrm{R}}$ & 1.17 & 1.84 & $9.99^{\mathrm{S}}$ & 1.27 & 2.98 & B \\
\hline & $5.37^{\mathrm{R}}$ & 1.18 & 2.69 & $4.17^{\mathrm{R}}$ & 1.21 & 2.27 & $4.55^{\mathrm{S}}$ & 1.22 & 1.53 & 4.90 & 1.00 & 0.00 & $4.72^{\mathrm{R}}$ & 1.09 & 1.01 & $4.24^{\mathrm{S}}$ & 1.18 & 1.36 & $\mathrm{C}$ \\
\hline 7 & $1.62^{\mathrm{S}}$ & 1.15 & 1.58 & $3.99^{S}$ & 1.34 & 1.81 & $2.44^{\mathrm{S}}$ & 1.90 & 3.47 & $11.69^{S}$ & 1.54 & 1.80 & $9.92^{\mathrm{S}}$ & 1.57 & 1.81 & 7.95 & 1.00 & 0.00 & A \\
\hline & $1.19^{S}$ & 1.30 & 2.29 & $1.16^{\mathrm{S}}$ & 1.19 & 1.12 & $0.85^{\mathrm{S}}$ & 1.24 & 1.55 & $1.33^{\mathrm{S}}$ & 1.27 & 1.75 & $1.37^{\mathrm{S}}$ & 1.27 & 1.62 & 2.08 & 1.00 & 0.00 & B \\
\hline & $1.21^{\mathrm{S}}$ & 1.13 & 1.28 & 1.11 & 1.00 & 0.00 & 0.83 & 1.00 & 0.00 & $0.94^{\mathrm{S}}$ & 1.14 & 1.03 & $0.88^{S}$ & 1.13 & 0.68 & 1.11 & 1.00 & 0.00 & C \\
\hline 8 & $3.41^{+}$ & 1.26 & 1.54 & $2.48^{+}$ & 1.53 & 2.85 & $2.74^{+}$ & 1.73 & 2.71 & $5.71^{+}$ & 1.24 & 1.30 & $4.98^{+}$ & 1.35 & 1.74 & 5.01 & 1.00 & 0.00 & A \\
\hline & $1.53^{+}$ & 1.18 & 1.64 & $1.43^{+}$ & 1.26 & 2.27 & $1.45^{+}$ & 1.41 & 1.99 & $1.45^{+}$ & 1.12 & 0.90 & $1.50^{+}$ & 1.21 & 1.53 & 2.22 & 1.00 & 0.00 & B \\
\hline & $1.68^{+}$ & 1.13 & 1.28 & $1.44^{+}$ & 1.16 & 1.30 & $1.42^{+}$ & 1.15 & 1.22 & $1.08^{+}$ & 1.09 & 0.28 & $1.12^{+}$ & 1.15 & 0.62 & 1.29 & 1.00 & 0.00 & C \\
\hline 9 & $3.36^{\mathrm{S}}$ & 1.16 & 1.42 & $1.59^{\mathrm{S}}$ & 1.15 & 1.06 & $1.53^{\mathrm{S}}$ & 1.20 & 1.59 & $5.46^{\mathrm{S}}$ & 1.25 & 1.68 & $4.31^{\mathrm{S}}$ & 1.12 & 1.57 & 4.99 & 1.00 & 0.00 & A \\
\hline & $0.84^{\mathrm{S}}$ & 1.16 & 1.16 & $0.72^{\mathrm{S}}$ & 1.14 & 0.87 & $0.77^{s}$ & 1.12 & 0.82 & $1.00^{\mathrm{S}}$ & 1.21 & 1.41 & $0.96^{S}$ & 1.20 & 1.30 & 1.57 & 1.00 & 0.00 & B \\
\hline & $1.00^{\mathrm{S}}$ & 1.08 & 0.55 & 0.88 & 1.00 & 0.00 & 1.01 & 1.00 & 0.00 & $0.69^{\mathrm{S}}$ & 1.13 & 0.66 & $0.69^{S}$ & 1.11 & 0.45 & 0.93 & 1.00 & 0.00 & C \\
\hline 10 & $2.77^{+}$ & 1.08 & 0.99 & 2.54 & 1.00 & 0.00 & 2.17 & 1.00 & 0.00 & 5.31 & 1.00 & 0.00 & 4.92 & 1.00 & 0.00 & 5.19 & 1.00 & 0.00 & A \\
\hline & 1.90 & 1.00 & 0.00 & $1.71^{-}$ & 1.11 & 1.00 & $1.57^{-}$ & 1.05 & 0.49 & $2.32^{-}$ & 1.06 & 0.30 & $2.51^{-}$ & 1.09 & 0.81 & $3.00^{-}$ & 1.14 & 1.66 & B \\
\hline & 2.05 & 1.00 & 0.00 & $1.67^{-}$ & 1.11 & 1.14 & $1.50^{-}$ & 1.05 & 0.48 & $1.80^{-}$ & 1.08 & 0.84 & $1.92^{-}$ & 1.10 & 0.99 & $1.84^{-}$ & 1.09 & 1.32 & C \\
\hline 11 & $7.97^{\mathrm{S}}$ & 1.28 & 1.88 & $5.68^{\mathrm{S}}$ & 1.42 & 1.88 & $3.88^{\mathrm{S}}$ & 1.59 & 1.98 & $23.44^{\mathrm{S}}$ & 1.35 & 1.66 & $17.55^{\mathrm{S}}$ & 1.45 & 2.07 & 18.00 & 1.00 & 0.00 & A \\
\hline & $1.94^{\mathrm{S}}$ & 1.24 & 2.12 & $1.66^{\mathrm{S}}$ & 1.27 & 1.81 & $1.30^{\mathrm{S}}$ & 1.20 & 1.54 & $1.88^{\mathrm{S}}$ & 1.36 & 1.81 & $2.06^{\mathrm{S}}$ & 1.39 & 1.90 & 3.43 & 1.00 & 0.00 & B \\
\hline & $2.10^{S}$ & 1.09 & 1.24 & $1.52^{\mathrm{S}}$ & 1.11 & 1.02 & 1.24 & 1.00 & 0.00 & $1.43^{\mathrm{S}}$ & 1.09 & 1.06 & $1.80^{\mathrm{S}}$ & 1.40 & 2.21 & 1.71 & 1.00 & 0.00 & $C$ \\
\hline 12 & $39.07^{+}$ & 1.17 & 1.70 & $26.39^{+}$ & 1.21 & 1.54 & $33.77^{-}$ & 1.08 & 0.33 & Retentiont & ime $>1$ & $20 \mathrm{~min}$ & Retention & ime $>1$ & $20 \mathrm{~min}$ & Retentior & ime $>1$ & $20 \mathrm{~min}$ & A \\
\hline & $18.11^{+}$ & 1.11 & 1.42 & $13.61^{+}$ & 1.19 & 1.78 & $15.20^{-}$ & 1.04 & 0.11 & $27.69^{+}$ & 1.11 & 1.14 & $26.03^{+}$ & 1.18 & 1.90 & Retentio & time $>1$ & $20 \mathrm{~min}$ & $B$ \\
\hline & $15.69^{+}$ & 1.13 & 1.95 & $11.99^{+}$ & 1.16 & 1.87 & 13.00 & 1.00 & 0.00 & $15.53^{+}$ & 1.14 & 1.57 & $13.64^{+}$ & 1.19 & 2.15 & 16.44 & 1.00 & 0.00 & C \\
\hline 13 & $45.80^{+}$ & 1.08 & 0.68 & Retention & ime $>1$ & $20 \mathrm{~min}$ & $32.52^{-}$ & 1.05 & 0.32 & Retentiont & ime $>1$ & $20 \mathrm{~min}$ & Retention & ime $>1$ & $20 \mathrm{~min}$ & Retention & ime $>1$ & $20 \mathrm{~min}$ & $\mathrm{~A}$ \\
\hline & $18.40^{+}$ & 1.11 & 1.36 & $12.55^{+}$ & 1.20 & 1.84 & $15.76^{-}$ & 1.03 & 0.14 & $28.04^{+}$ & 1.10 & 1.05 & $25.00^{+}$ & 1.17 & 1.74 & Retentic & ime $>1$ & $20 \mathrm{~min}$ & $\mathrm{~B}$ \\
\hline & $16.24^{+}$ & 1.12 & 1.79 & $11.88^{+}$ & 1.17 & 1.94 & 12.73 & 1.00 & 0.00 & $15.07^{+}$ & 1.13 & 1.56 & $13.51^{+}$ & 1.19 & 2.11 & 15.78 & 1.00 & 0.00 & C \\
\hline 14 & $1.43^{\mathrm{R}}$ & 1.10 & 0.28 & $1.17^{\mathrm{S}}$ & 1.23 & 0.94 & $1.97^{S}$ & 1.16 & 0.69 & 2.55 & 1.00 & 0.00 & $1.96^{\mathrm{R}}$ & 1.15 & 0.38 & 1.60 & 1.00 & 0.00 & A \\
\hline & $1.03^{\mathrm{R}}$ & 1.07 & 0.17 & $0.86^{\mathrm{S}}$ & 1.17 & 0.56 & $1.48^{\mathrm{S}}$ & 1.13 & 0.60 & 1.17 & 1.00 & 0.00 & 1.13 & 1.00 & 0.00 & 0.89 & 1.00 & 0.00 & B \\
\hline & $1.21^{\mathrm{R}}$ & 1.05 & 0.14 & $0.93^{S}$ & 1.14 & 0.69 & $1.39^{\mathrm{S}}$ & 1.10 & 0.59 & 1.19 & 1.00 & 0.00 & 0.98 & 1.00 & 0.00 & 0.89 & 1.00 & 0.00 & $\mathrm{C}$ \\
\hline 15 & $2.74^{\mathrm{S}}$ & 1.11 & 0.94 & $1.93^{\mathrm{S}}$ & 1.17 & 1.13 & $1.70^{\mathrm{S}}$ & 1.13 & 0.66 & $3.92^{\mathrm{S}}$ & 1.05 & 0.10 & $3.57^{\mathrm{S}}$ & 1.15 & 0.74 & 5.10 & 1.00 & 0.00 & A \\
\hline & $1.51^{\mathrm{S}}$ & 1.08 & 0.61 & 1.31 & 1.00 & 0.00 & 1.13 & 1.00 & 0.00 & 1.25 & 1.00 & 0.00 & 1.40 & 1.00 & 0.00 & 2.54 & 1.00 & 0.00 & B \\
\hline & 1.64 & 1.00 & 0.00 & 1.53 & 1.00 & 0.00 & $1.08^{\mathrm{R}}$ & 1.10 & 0.83 & 0.98 & 1.00 & 0.00 & 1.07 & 1.00 & 0.00 & $1.44^{\mathrm{R}}$ & 1.09 & 0.87 & C \\
\hline 16 & $47.31^{S}$ & 1.26 & 1.75 & $38.59^{S}$ & 1.52 & 2.71 & $22.37^{S}$ & 1.09 & 0.44 & Retentiont & ime $>1$ & $20 \mathrm{~min}$ & Retention & ime $>1$ & $20 \mathrm{~min}$ & Retention & time $>1$ & $20 \mathrm{~min}$ & $\mathrm{~A}$ \\
\hline & $15.18^{S}$ & 1.07 & 0.68 & $13.70^{\mathrm{S}}$ & 1.30 & 2.29 & 11.58 & 1.00 & 0.00 & $11.71^{\mathrm{S}}$ & 1.16 & 1.23 & $18.22^{\mathrm{S}}$ & 1.20 & 1.51 & $13.03^{\mathrm{S}}$ & 1.24 & 1.55 & B \\
\hline & $20.03^{\mathrm{R}}$ & 1.10 & 1.41 & $18.21^{\mathrm{S}}$ & 1.16 & 1.62 & 13.35 & 1.00 & 0.00 & 11.01 & 1.00 & 0.00 & $14.49^{S}$ & 1.06 & 0.29 & $5.42^{\mathrm{S}}$ & 1.16 & 1.97 & C \\
\hline 17 & $9.37^{\mathrm{S}}$ & 1.02 & 0.15 & $14.40^{\mathrm{S}}$ & 1.19 & 0.73 & $16.43^{S}$ & 1.14 & 0.48 & Retentiont & ime $>1$ & $20 \mathrm{~min}$ & Retention & ime $>1$ & $20 \mathrm{~min}$ & Retention & time $>1$ & $20 \mathrm{~min}$ & $\mathrm{~A}$ \\
\hline & 7.33 & 1.00 & 0.00 & $6.13^{S}$ & 1.09 & 0.34 & $6.47^{\mathrm{R}}$ & 1.25 & 1.76 & $9.68^{\mathrm{S}}$ & 1.14 & 0.93 & $9.07^{\mathrm{S}}$ & 1.16 & 0.98 & 11.31 & 1.00 & 0.00 & B \\
\hline & 6.50 & 1.00 & 0.00 & 5.33 & 1.00 & 0.00 & $5.56^{\mathrm{R}}$ & 1.29 & 2.59 & 6.30 & 1.00 & 0.00 & 5.35 & 1.00 & 0.00 & $4.67^{\mathrm{R}}$ & 1.10 & 0.96 & $C$ \\
\hline 18 & 8.34 & 1.00 & 0.00 & $5.48^{\mathrm{S}}$ & 1.05 & 0.10 & $3.75^{\mathrm{S}}$ & 1.22 & 1.10 & 12.70 & 1.00 & 0.00 & 8.47 & 1.00 & 0.00 & 8.97 & 1.00 & 0.00 & A \\
\hline & 1.94 & 1.00 & 0.00 & 1.21 & 1.00 & 0.00 & $1.93^{\mathrm{S}}$ & 1.17 & 1.04 & 2.52 & 1.00 & 0.00 & $1.95^{\mathrm{S}}$ & 1.15 & 0.50 & 3.03 & 1.00 & 0.00 & B \\
\hline & 1.73 & 1.00 & 0.00 & 1.13 & 1.00 & 0.00 & $1.91^{\mathrm{S}}$ & 1.11 & 0.81 & 2.09 & 1.00 & 0.00 & 1.50 & 1.00 & 0.00 & 1.95 & 1.00 & 0.00 & C \\
\hline 19 & $15.982^{S, 3 R}$ & 2.64 & 6.81 & $10.79^{2 S, 3 R}$ & 3.28 & 6.66 & $10.30^{2 S, 3 R}$ & 4.32 & 8.05 & $19.63^{2 S, 3 R}$ & 1.59 & 3.28 & $10.85^{2 \mathrm{~S}, 3 \mathrm{R}}$ & 2.12 & 5.18 & $13.20^{2 \mathrm{~S}, 3 \mathrm{R}}$ & 3.57 & 6.24 & A \\
\hline & $4.022^{\mathrm{S}, 3 \mathrm{R}}$ & 1.91 & 6.24 & $2.45^{2 S, 3 R}$ & 1.93 & 4.97 & $2.08^{2 S, 3 R}$ & 2.42 & 7.75 & $4.01^{2 \mathrm{~S}, 3 \mathrm{R}}$ & 1.34 & 2.49 & $4.67^{2 \mathrm{~S}, 3 \mathrm{R}}$ & 1.68 & 3.79 & $2.20^{2 \mathrm{~S}, 3 \mathrm{R}}$ & 1.21 & 2.60 & B \\
\hline & $3.93^{2 \mathrm{~S}, 3 \mathrm{R}}$ & 1.89 & 6.36 & $2.78^{2 \mathrm{~S}, 3 \mathrm{R}}$ & 2.48 & 6.06 & $3.04^{2 S, 3 R}$ & 3.12 & 11.90 & $4.11^{2 \mathrm{~S}, 3 \mathrm{R}}$ & 1.32 & 2.35 & $2.23^{2 S, 3 R}$ & 1.49 & 3.56 & $2.42^{2 \mathrm{~S}, 3 \mathrm{R}}$ & 2.12 & 5.54 & C \\
\hline
\end{tabular}

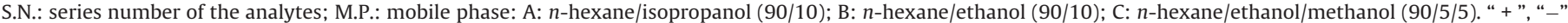
"R", "S" and "(2S,3R)" at the superscript of $k_{1}$ refer to the optical rotation or configuration of the first-eluted enantiomer.

Flow rate: $1 \mathrm{ml} \mathrm{min}^{-1}$.

Dectetion temperature: $25^{\circ} \mathrm{C}$. 


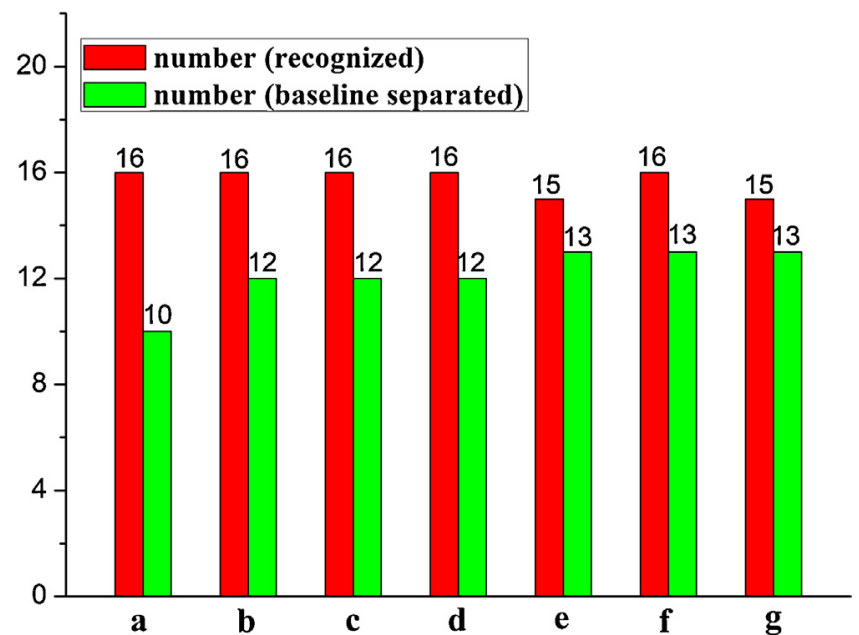

Fig. 7. Numbers of racemates recognized and baseline separated by CSP5 before and after being flushed by various organic solvents as the eluents. (a) initial enantioseparation results of CSP5; (b) after working for $100 \mathrm{~h}$ since the first-time enantioseparation and then being placed aside for two months; (c) after being flushed with ethyl acetate (100\%); (d) after being flushed with chloroform (100\%); (e) after being flushed with THF/n-hexane (50/50); (f) after being flushed with THF/n-hexane (60/40); (g) after being flushed with THF/n-hexane (70/30). Enantioseparation eluent for a-g: $n$-hexane/ethanol (90/10); flow rate: $1 \mathrm{ml} \mathrm{min}^{-1}$; dectetion temperature: $25^{\circ} \mathrm{C}$.

The chiral recognition abilities of the prepared CSPs were evaluated by the enantioseparation of nineteen chiral analytes by HPLC in the eluents of $n$-hexane/isopropanol (90/10), $n$-hexane/ethanol (90/10) and $n$-hexane/methanol/ethanol (90/5/5), respectively. The structures of the tested chiral analytes are shown in Fig. 5. The enantioseparation results of the nineteen racemic compounds on the prepared CSPs are summarized and shown in Table 1 and Figs. S7-S9. It was found that the enantioseparation ability of the chitosan derivatives based CSPs depended very much on the position of methyl substituent and the substitution at 3position on the phenyl group seemed to be the most preferable. Among these newly developed CSPs, except for chitosan bis(2methylphenylcarbamate)-(isobutyrylamide)-derived CSP (CSP6), all other CSPs exhibited satisfactory chiral recognition abilities for most of the racemates with some chiral compounds being baseline separated. Take CSP1 for example, among the nineteen chiral analytes, eighteen racemates could be chirally recognized and nine racemates could be baseline separated by using $n$-hexane/isopropanol as the mobile phase. Representative chromatograms for the effective enantioseparation of some racemates by the prepared CSPs are shown in Fig. 6. When compared with the structures of chitosan bis(phenylcarbamate)(urea) derivatives [22], it is found that there is an alkyl amide rather than phenyl urea at 2-position of the chitosan derivatives in the present study, and these newly developed CSPs based on chitosan bis(methylphenylcarbamate)-(isobutyrylamide) also exhibited satisfactory chiral resolution abilities, indicating that the aromatic ring at 2-position of chitosan derivatives may not be necessary for chiral recognition. This conclusion will provide a useful consideration to design new types of chitosan derivatives based CSPs for enantiomer separations.

\subsubsection{Influence of eluent composition on enantioseparations}

Influence of eluent composition on enantioseparations by the prepared CSPs was investigated in detail. As shown in Table 1 and Figs. S7-S9, more effective enantioseparations with higher separation factors, and with more racemates being recognized and baseline separated, could be achieved in the eluents of $n$ hexane/isopropanol and $n$-hexane/ethanol than in the eluent of

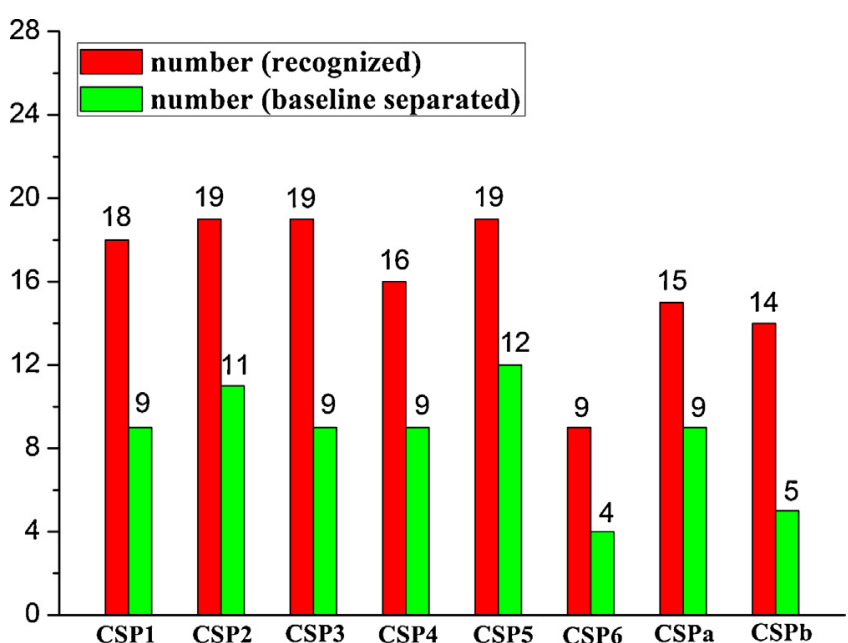

Fig. 8. Total numbers of racemates recognized or baseline separated by CSPs1-6, $\mathrm{CSPa}$ and $\mathrm{CSPb}$ in the mobile phases containing various alcohols.

n-hexane/methanol/ethanol. Take CSP2 for example, the chiral recognition ability in different eluents was in accordance with the following order: $n$-hexane/ethanol $>n$-hexane/isopropanol $>n$ hexane/methanol/ethanol (Figs. S7-S9). As can be seen in Table 1, racemate 3 could be separated by CSP2 only in ethanol-containing mobile phase, while racemates 15 and 18 could be separated only in isopropanol-containing mobile phase. Although racemate 8 was separated by CSP2 in isopropanol-, ethanoland (methanol+ethanol)-containing mobile phases, respectively. However, the resolutions decreased in sequence. On the other hand, methanol was found to be superior to ethanol and isopropanol as the eluent for the enantiomeric separation of racemate $\mathbf{1 2}$ (Fig. 6a).

\subsubsection{Influence of the substituent group on the phenyl moieties of the chitosan derivatives on enantioseparations}

Okamoto et al. once reported that the introduction of either electron-donating or electron-withdrawing substituents at 3- or 4-position onto the phenyl moieties tended to improve the chiral recognition ability of cellulose phenylcarbamate derivatives [13]. The similar trends could also be found for the chitosan derivatives [22,25]. As shown in Table 1 and Figs. S7-S9, CSPs1-3 and CSP5 with substituents at 3- or 4-position on the phenyl moieties could provide more effective enantioseparation for the tested racemates, while CSP4 and CSP6 without substituent or with a methyl group at the 2-position on the corresponding phenyl moieties exhibited comparatively poorer chiral recognition ability. The chromatographic performance of the CSPs with different substituents on the phenyl moieties was in accordance with the following order: 3-methyl > 4-methyl > no substituent $>2$-methyl. Regarding the chitosan derivatives with and without the substituent of chloro group on the phenyl moieties, the chiral recognition abilities were in the order of 4-methyl > 3-chloro-4-methyl by comparing the separation performance.

\subsubsection{Influence of the structure of racemate on enantioseparations}

The choice of tested racemates is always very critical for the evaluation of the chromatographic performance of CSPs [35]. In the present work, most of the nineteen racemic analytes are drugs or the intermediates for drugs. Racemates including $\mathbf{1}, \mathbf{2}, \mathbf{4}, \mathbf{6}, \mathbf{7}, \mathbf{8}, \mathbf{9}, 11$ and 19 could be resolved better by the prepared CSPs. These racemic analytes contain $\mathrm{C}=\mathrm{O}, \mathrm{S}=\mathrm{O}$, amido, ureido or $\mathrm{N}$-heterocyclic group, which can form dipole-dipole interactions, hydrogen bonds and $\pi-\pi$ interactions with the CSPs, thus enabling them to be more easily recognized since more interactions were involved during 


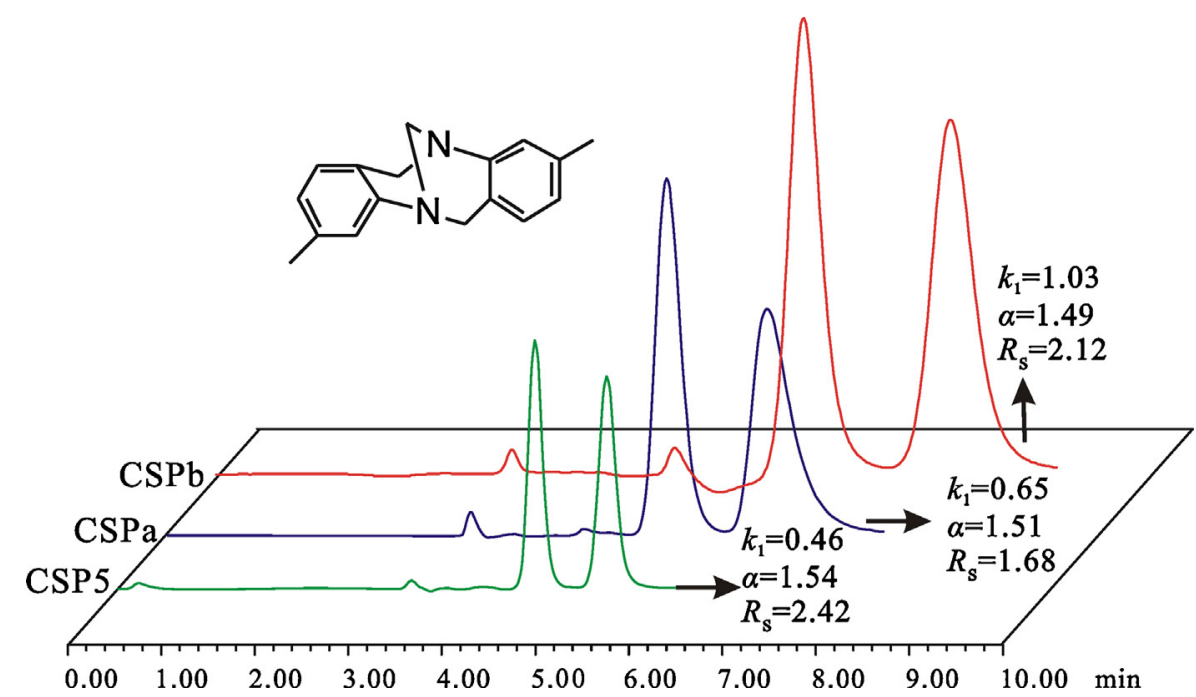

Fig. 9. Chromatograms for the resolution of racemate $\mathbf{1}$ in $n$-hexane/isopropanol on CSP5, CSPa and CSPb.

enantioseparation. The results revealed that these chitosan derivatives based CSPs were suitable for the enantioseparation of some chiral drugs. Compared with racemates $\mathbf{7}$ and $\mathbf{9}$, racemates $\mathbf{1 1}$ and $\mathbf{1 6}$ were retained longer. The main distinction of the two groups of compounds is that racemates $\mathbf{1 1}$ and $\mathbf{1 6}$ possess electron-donating group $\left(-\mathrm{OCH}_{3}\right)$ at the para-position on the benzene ring, and two nitro groups are attached on another phenyl group for racemate $\mathbf{1 6}$, which resulted in stronger interactions between the analytes and the CSPs, therefore leading to be eluted latter.

\subsection{Eluents tolerance of the CSPS}

It is well known that traditional coated-type chiral packing materials based on cellulose and amylose derivatives are not usually used in the eluents containing chloroform, ethyl acetate etc., and cannot be used in tetrahydrofuran (THF) containing eluent in particular, because the derivatives can highly swell or even dissolve in these solvents. However, sometimes it is strongly desired to use these solvents in the eluents since the addition of these organic modifiers may improve the separation performance and also solve the problem of poor solubility of a sample for preparative separation [36,37]. Therefore, the development of desirable CSPs which possess satisfactory chiral separation ability and meanwhile can be analyzed with a wide range of eluents is very important and meaningful. In order to address the problem of dissolution and swelling in some unusual eluents, some immobilized polysaccharide-derived CSPs were developed [38-42]. Chloroform, ethyl acetate and THF were previously employed as the compositions of mobile phases for immobilized-type CSPs (Chiralpak IA, Chiralpak IB and Chiralpak IC), and satisfactory separations were achieved in the presence of these organic modifiers $[43,44]$. In the present study, the synthesized chitosan derivatives showed much lower solubility in the above-mentioned solvents in comparison with cellulose and amylose derivatives, hence, the prepared CSPs can work in the unusual eluents consisting of these solvents. CSP5 with the best chiral recognition ability was selected as the stationary phase for the investigation of solvent tolerance. After CSP5 worked for $100 \mathrm{~h}$ since the first-time enantioseparation evaluation (Fig. 7a), and then was placed aside for two months, the same enantioseparations were tested again with the mobile phase of $n$-hexane/ethanol (90/10) (Fig. 7b), in which the best separation performance was initially gained. As shown in Table S1, racemate $\mathbf{9}$ initially was not baseline separated $\left(R_{\mathrm{S}}=1.30\right.$, Table $\left.\mathrm{S} 1(\mathrm{a})\right)$, while the baseline separation was gained $\left(R_{S}=1.51\right.$, Table S1(b)) after CSP5 had been analyzed for $100 \mathrm{~h}$ and had been put aside for two months; racemate 19 was retained for a long time initially (retention time $>120 \mathrm{~min}$, Table $\mathrm{S} 1(\mathrm{a}))$, and then was baseline separated $\left(R_{\mathrm{S}}=3.79\right.$, Table S1(b)); however, racemate $\mathbf{1 8}$ could not be separated again that was only one exceptional example; some racemates were separated again with a slight increase in resolution and few with a slight decrease; the rest racemates were separated almost with unchanged resolution. The overall separation ability of CSP5 did not changed significantly. Afterwards, the eluents tolerance of CSP5 was investigated as follows: CSP5 was first flushed by a test eluent with a flow rate of $1.0 \mathrm{ml} \mathrm{min}^{-1}$ at $25^{\circ} \mathrm{C}$ for $7 \mathrm{~h}$, and then the enantioseparation was evaluated in the mobile phase of $n$-hexane/ethanol $(90 / 10)$. By the same way, CSP5 was flushed again with another eluent and enantioseparation evaluation was repeated again. The flushing and evaluation were carried on five times in total. The test results were summarized and shown in Table S1 and Fig. 7. Herein, the eluents tolerance of CSP5 was tested in a different way compared to other reported works [22]. Specifically, this tolerance test was conducted in a more extreme case with chloroform $(100 \%)$ (Fig. 7c), ethyl acetate (100\%) (Fig. 7d) and THF/n-hexane (50/50, $60 / 40,70 / 30$ ) (Fig. 7e-g) as the test eluents in sequence rather than by adding a small quantity of these solvent into the mobile phases. Racemate 10 was separated with a low resolution $\left(R_{\mathrm{S}}=0.65\right.$, Table S1(b)) before CSP5 was flushed with the test eluents. The resolution nearly did not change after CSP5 was flushed with pure ethyl acetate $\left(R_{\mathrm{S}}=0.66\right.$, Table $\left.\mathrm{S} 1(\mathrm{c})\right)$, and slightly became lower $\left(R_{\mathrm{S}}=0.41\right.$, Table S1(d)) after CSP5 was flushed with pure chloroform. After CSP5 was flushed by THF/ $n$-hexane (50/50), racemate $\mathbf{1 0}$ could not be resolved. However, most of the racemates were separated with improved resolutions at different extent after CSP5 was flushed with various eluents. For instance, the separation factor and resolution of racemate $\mathbf{1 7}$ exhibited a slightly increasing tendency after CSP5 was flushed with the test eluents and finally baseline enantioseparation was achieved $\left(R_{\mathrm{S}} \geq 1.65\right.$, Table $\left.\mathrm{S} 1(\mathrm{e}-\mathrm{g})\right)$. As shown in Fig. 7 , the numbers of racemates, which were chirally recognized and baseline separated by CSP5 before and after being flushed by various eluents, did not change significantly.

Generally, CSP5 still exhibited powerful enantioseparation capability, although it was flushed with chloroform (100\%), ethyl acetate (100\%) and even THF/n-hexane (70/30). In contrast to the coated-type CSPs of CDMPC and ADMPC which are soluble or highly swell in these eluents, CSP5 can work in the above mentioned eluents. Therefore, the application of the prepared chitosan derivatives based CSPs perhaps can address the problem concerning dissolu- 
tion and high swelling of coated-type CSPs prepared from cellulose and amylose derivatives in some eluents, further broadening the possibility of eluent choice.

\subsection{Enantioseparation comparison of the prepared CSPs with the CDMPC- and ADMPC-based CSPS}

The chiral recognition abilities of the prepared CSPs were compared with those of ADMPC- and CDMPC-based CSPs, i.e., CSPa and $\mathrm{CSPb}$, respectively. The overall separation performances of the above mentioned CSPs in different eluents are summarized in Table 1 and Table S2. Fig. 8 displays the total numbers of the analytes recognized or baseline separated by each CSP in all the three alcohols containing eluents. Based on the presented results, we found that the chiral recognition ability of all the chitosan derivatives based CSPs (except for CSP6) for the tested racemates was better than that of CSPa and CSPb as more racemates were recognized and baseline separated. Above all, relatively more satisfactory separation performances could be achieved by CSP2, CSP3 and CSP5 with all the chiral analytes being recognized, and CSP5 exhibited the best enantioseparation.

In the eluent of $n$-hexane/isopropanol (90/10), there were nine racemates could be baseline separated by CSP1, CSP2 and CSP5, while only four by both CSPa and CSPb. Racemates $1,2,4,5,6,11$ and 19 were found to be more effectively resolved by CSP5 with higher $R_{\mathrm{S}}$ values compared with CSPa and $\mathrm{CSPb}$. And racemate $\mathbf{1 4}$ could only be chirally recognized by CSP5. Enantioseparations of racemate $\mathbf{1}$ by CSP5, CSPa and $\mathrm{CSPb}$ were specifically discussed. As shown in Fig. 9, the $k_{1}$, $\alpha$ and $R_{\mathrm{S}}$ values for racemate 1 decreased in the following order: $k_{1}(\mathrm{CSPb})>k_{1}(\mathrm{CSPa})>k_{1}(\mathrm{CSP} 5), \alpha(\mathrm{CSP} 5)>\alpha(\mathrm{CSPa})>\alpha(\mathrm{CSPb})$ and $R_{\mathrm{S}}(\mathrm{CSP} 5)>R_{\mathrm{S}}(\mathrm{CSPb})>R_{\mathrm{S}}(\mathrm{CSPa})$, respectively. Therefore, racemate 1 was more satisfactorily separated by CSP5 than by CSPa and $\mathrm{CSPb}$ under the same chromatographic conditions.

As discussed above, we could safely draw the conclusion that most of the resultant chitosan derivatives based CSPs exhibited excellent separation performance. Notably, when compared with the separation performance of CSP2 with CSPa or CSPb, which all bear the same carbamate at 3- and 6-positions on the glucose unit, it was found that the enantioseparation ability of CSP2 was even superior to that of ADMPC- and CDMPC-based CSPs for the tested racemates.

\section{Conclusion}

In this study, a series of new CSPs were prepared by coating chitosan isobutyrylamide derivatives onto 3-aminopropyl silica gel, whose chiral recognition abilities were evaluated by HPLC with nineteen racemates. The results demonstrated that the eluent composition, the substituent on the phenyl moieties of chitosan derivatives, as well as the structure of racemates significantly affected the enantioseparations. Due to the fact that much lower solubility of the chitosan derivatives was observed, the eluent tolerance of the prepared CSPs was found to be more satisfactory in comparison with the coated-type CSPs based on cellulose and amylose derivatives, broadening the possibility of eluent choice. In addition, most of the chitosan isobutyrylamide derivatives based CSPs exhibited excellent enantioseparation performance for some chiral drugs, indicating their promising application prospects. Relative to CDMPC and ADMPC, there is an alkyl amide rather than 3,5-dimethylphenyl carbamate at 2-position of the chitosan derivatives presented in this study, implying that the aromatic ring at 2-position of glucose unit may not be necessary for chiral recognition. This will provide a new consideration to design chitosan derivatives based CSPs for enantiomer separations.

\section{Acknowledgments}

The authors express their thanks to the support of the National Natural Science Foundation of China (No. 51373127).

\section{Appendix A. Supplementary data}

Supplementary data associated with this article can be found, in the online version, at http://dx.doi.org/10.1016/j.chroma.2016.02. 053.

\section{References}

[1] J. Shen, Y. Okamoto, Efficient separation of enantiomers using stereoregular chiral polymers, Chem. Rev. 16 (2016) 1094-1138.

[2] B. Chankvetadze, Recent developments on polysaccharide-based chiral stationary phases for liquid-phase separation of enantiomers, J. Chromatogr. A 1269 (2012) 26-51.

[3] M. Tang, J. Zhang, S. Zhuang, W. Liu, Development of chiral stationary phases for high-performance liquid chromatographic separation, TrAC-Trend Anal. Chem. 39 (2012) 180-194.

[4] J. Shen, T. Ikai, Y. Okamoto, Synthesis and application of immobilized polysaccharide-based chiral stationary phases for enantioseparation by highperformance liquid chromatography, J. Chromatogr. A 1363 (2014) 51-61.

[5] Y. Okamoto, T. Ikai, Chiral HPLC for efficient resolution of enantiomers, Chem. Soc. Rev. 37 (2008) 2593-2608.

[6] B. Chankvetadze, T. Ikai, C. Yamamoto, Y. Okamoto, High-performance liquid chromatographic enantioseparations on monolithic silica columns containing a covalently attached 3,5-dimethylphenylcarbamate derivative of cellulose, J. Chromatogr. A 1042 (2004) 55-60.

[7] Z.Q. Wang, J.D. Liu, W. Chen, Z.W. Bai, Enantioseparation characteristics of biselector chiral stationary phases based on derivatives of cellulose and amylose, J. Chromatogr. A 1346 (2014) 57-68.

[8] I. Matarashvili, I. Shvangiradze, L. Chankvetadze, S. Sidamonidze, N. Takaishvili, T. Farkas, B. Chankvetadze, High-performance liquid chromatographic separations of stereoisomers of chiral basic agrochemicals with polysaccharide-based chiral columns and polar organic mobile phases, J. Sep. Sci. 38 (2015) 4173-4179.

[9] E. Yashima, Polysaccharide-based chiral stationary phases for highperformance liquid chromatographic enantioseparation, J. Chromatogr. A 906 (2001) 105-125.

[10] P. López-Ram-de-Víu, J.A. Gálvez, M.D. Díaz-de-Villegas, High-performance liquid chromatographic enantioseparation of unusual amino acid derivatives with axial chirality on polysaccharide-based chiral stationary phases, J. Chromatogr. A 1390 (2015) 78-85

[11] Y. Okamoto, Y. Kaida, Resolution by high-performance liquid chromatography using polysaccharide carbamates and benzoates as chiral stationary phases, J. Chromatogr. A 666 (1994) 403-419.

[12] A. Ishikawa, T. Shffiata, Cellulosic chiral stationary phase under reversed-phase condition, J. Liq. Chromatogr. Rel. Technol. 16 (1993) 859-878.

[13] Y. Okamoto, M. Kawashima, K. Hatada, Chromatographic resolution: XI. Controlled chiral recognition of cellulose triphenylcarbamate derivatives supported on silica gel, J. Chromatogr. A 363 (1986) 173-186.

[14] Y. Okamoto, M. Kawashima, K. Hatada, Chromatographic resolution. 7. Useful chiral packing materials for high-performance liquid chromatographic resolution of enantiomers: phenylcarbamates of polysaccharides coated on silica gel, J. Am. Chem. Soc. 106 (1984) 5357-5359.

[15] S. Rizzo, S. Menta, C. Faggi, M. Pierini, R. Cirilli, Influence of the nature of alkyl substituents on the high-performance liquid chromatography enantioseparation and retention of new atropisomeric $11^{\prime}$ '-bibenzimidazole derivatives on amylose tris (3,5-dimethylphenylcarbamate) chiral stationary phase, J. Chromatogr. A 1363 (2014) 128-136.

[16] T. Ikai, C. Yamamoto, M. Kamigaito, Y. Okamoto, Immobilization of polysaccharide derivatives onto silica gel: facile synthesis of chiral packing materials by means of intermolecular polycondensation of triethoxysilyl groups, J. Chromatogr. A 1157 (2007) 151-158.

[17] A.A. Younes, H. Ates, D. Mangelings, Y. Vander Heyden, A separation strategy combining three HPLC modes and polysaccharide-based chiral stationary phases, J. Pharmaceut. Biomed. 75 (2013) 74-85.

[18] X.C. Wang, J. Zhang, X.Q. Xu, W. Chen, Y.G. Yang, Z.W. Bai, Enantioseparation characteristics of chiral stationary phases based on the derivatives of cellulose and chitin, Anal. Methods 7 (2015) 2786-2793.

[19] Y. Okamoto, R. Aburatani, K. Hatada, M. Honda, N. Inotsume, M. Nakano, Optica resolution of dihydropyridine enantiomers by high-performance liquid chromatography using phenylcarbamates of polysaccharides as a chiral stationary phase, J. Chromatogr. A 513 (1990) 375-378.

[20] Y. Okamoto, J. Noguchi, E. Yashima, Enantioseparation on 3,5-dichloro- and 3,5-dimethylphenylcarbamates of polysaccharides as chiral stationary phases for high-performance liquid chromatography, React Funct. Polym. 37 (1998) $183-188$ 
[21] T. Ikai, Y, Okamoto, Structure control of polysaccharide derivatives for efficient separation of enantiomers by chromatography, Chem. Rev. 109 (2009) 6077-6101.

[22] L. Zhang, J. Shen, W. Zuo, Y. Okamoto, Synthesis of chitosan 3,6diphenylcarbamate-2-urea derivatives and their applications as chiral stationary phases for high-performance liquid chromatography, J. Chromatogr. A 1365 (2014) 86-93.

[23] S.H. Son, J. Jegal, Synthesis and characterization of the chiral stationary phase based on chitosan, J. Appl. Polym. Sci. 106 (2007) 2989-2996.

[24] A. Senso, L. Oliveros, C. Minguillón, Chitosan derivatives as chiral selectors bonded on allyl silica gel: preparation, characterisation and study of the resulting high-performance liquid chromatography chiral stationary phases, J. Chromatogr. A 839 (1999) 15-21.

[25] C. Yamamoto, M. Fujisawa, M. Kamigaito, Y. Okamoto, Enantioseparation using urea- and imide-bearing chitosan phenylcarbamate derivatives as chiral stationary phases for high-performance liquid chromatography, Chirality 20 (2008) 288-294.

[26] S.H. Son, J. Jegal, Synthesis and characterization of the chiral stationary phase based on chitosan, J. Appl. Polym. Sci. 106 (2007) 2989-2996.

[27] J.L. Chen, H.J. Syu, Immobilization of chitosan in sol-gel phases for chiral open-tubular capillary electrochromatography, Anal. Chim. Acta 718 (2012) $130-137$.

[28] X. Han, L. An, H. Cui, H. Li, W. Liu, Enantioseparation using chitosan tris (3-chlorophenylcarbamate) as a chiral stationary phase for HPLC, Chromatographia 73 (2011) 1043-1047.

[29] Z. Lü, P. Zhang, L. Jia, Preparation of chitosan functionalized monolithic silica column for hydrophilic interaction liquid chromatography, J. Chromatogr. A 1217 (2010) 4958-4964.

[30] Y. Liu, H.Zou, J. Haginaka, Preparation and evaluation of a novel chiral stationary phase based on covalently bonded chitosan for ligand-exchange chromatography, J. Sep. Sci. 29 (2006) 1440-1446.

[31] J.L. Chen, Molecularly bonded chitosan prepared as chiral stationary phases in open-tubular capillary electrochromatography: comparison with chitosan nanoparticles bonded to the polyacrylamide phase, Talanta 85 (2011) 2330-2338.

[32] J. Zhang, Z.Q. Wang, X.C. Wang, J.J. Zhang, Z.W. Bai, W. Chen, Enantioseparation characteristics of tadalafil and its intermediate on chitin derived chiral stationary phases, Analyst 140 (2015) 5593-5600
[33] M. Lavertu, Z. Xia, A. Serreqi, M. Berrada, A. Rodrigues, D. Wang, M. Buschmann, A. Gupta, A validated ${ }^{1} \mathrm{H}$ NMR method for the determination of the degree of deacetylation of chitosan, J. Pharmaceut. Biomed. 32 (2003) 1149-1158.

[34] M.R. Kasaai, Determination of the degree of $\mathrm{N}$-acetylation for chitin and chitosan by various NMR spectroscopy techniques: a review, Carbohydr. Polym. 79 (2010) 801-810.

[35] A. Novell, C. Minguillón, Octaproline, a conformationally flexible chiral selec tor in liquid chromatographic enantioseparation, J. Chromatogr. A 1363 (2014) 109-118.

[36] T. Ikai, C. Yamamoto, M. Kamigaito, Y. Okamoto, Immobilized polysaccharidebased chiral stationary phases for HPLC, Polym. J. 38 (2006) 91-108.

[37] C. Yamamoto, T. Hayashi, Y. Okamoto, S. Ohkubo, T. Kato, Direct resolution of C76 enantiomers by HPLC using an amylose-based chiral stationary phase, Chem. Commun. (2001) 925-926.

[38] A. Senso, L. Oliveros, C. Minguillón, Chitosan derivatives as chiral selectors bonded on allyl silica gel: preparation, characterisation and study of the resulting high-performance liquid chromatography chiral stationary phases, J. Chromatogr. A 839 (1999) 15-21.

[39] P. Franco, A. Senso, C. Minguillón, L. Oliveros, 3,5-Dimethylphenylcarbamates of amylose, chitosan and cellulose bonded on silica gel: comparison of their chiral recognition abilities as high-performance liquid chromatography chiral stationary phases, J. Chromatogr. A 796 (1998) 265-272.

[40] P. Franco, A. Senso, L. Oliveros, C. Minguillón, Covalently bonded polysaccharide derivatives as chiral stationary phases in high-performance liquid chromatography, J. Chromatogr. A 906 (2001) 155-170.

[41] K. De Klerck, Y. Vander Heyden, D. Mangelings, Pharmaceutical-enantiomers resolution using immobilized polysaccharide-based chiral stationary phases in supercritical fluid chromatography, J. Chromatogr. A 1328 (2014) 85-97.

[42] R. Geryk, K. Kalíková,J. Vozka, D. Plecitá, M.G. Schmid, E. Tesařová, Enantioselective potential of chiral stationary phases based on immobilized polysaccharides in reversed phase mode, J. Chromatogr. A 1363 (2014) 155-161.

[43] T. Zhang, M. Schaeffer, P. Franco, Optimization of the chiral separation of a Ca-sensitizing drug on an immobilized polysaccharide-based chiral stationary phase: case study with a preparative perspective, J. Chromatogr. A 1083 (2005) 96-101.

[44] T. Zhang, D. Nguyen, P. Franco, Enantiomer resolution screening strategy using multiple immobilised polysaccharide-based chiral stationary phases, J. Chromatogr. A 1191 (2008) 214-222. 